\title{
SiO MASERS IN VARIABLE STARS
}

\author{
GuY N. Blair \\ The University of Texas at Austin and the Netherlands Foundation for Radio Astronomy \\ AND \\ DALE F. DiCKINSON \\ Center for Astrophysics, Harvard College Observatory and the Smithsonian Astrophysical Observatory \\ Received 1976 November 29
}

\begin{abstract}
Eight new long-period variable stars were found with maser emission from the first excited vibrational state of silicon monoxide. One of these, W Andromedae, is a spectral type S star, the second $\mathrm{S}$ star known to show $\mathrm{SiO}$ emission. A second, T Cephei, is a borderline case between $\mathrm{M}$ and $\mathrm{S}$. Although $\mathrm{H}_{2} \mathrm{O}$ and $\mathrm{OH}$ maser emission is commonly found in the $\mathrm{SiO}$ stars, a search of $26 \mathrm{~S}$-type stars for water has been negative, suggesting that the effect is real and arises from a chemical difference between the $\mathrm{S}$ stars and those of spectral type M. This may be a result of the high $\mathrm{C} / \mathrm{O}$ ratio in spectral type $\mathrm{S}$.

A new source of ground-state $\mathrm{SiO}$ emission was found in NGC 2264. This is the first thermal
\end{abstract} $\mathrm{SiO}$ emission observed in a molecular cloud outside of Sgr B2 and Orion A.

Subject headings: masers - radio sources: lines - stars: long-period variables - stars: S-type

\section{INTRODUCTION}

Maser emission from vibrationally excited $\mathrm{SiO}$ was first observed by Snyder and Buhl (1974) in Orion A; subsequently, $20 \mathrm{SiO}$ maser stars were found by Kaifu, Buhl, and Snyder (1975), and Snyder and Buhl (1975). These stars are Mira-type and semiregular long-period variables, and all but three have water-vapor maser emission at $22 \mathrm{GHz}$. One of these three, $\chi$ Cyg, is a spectral type $\mathbf{S}$ star; such stars are thought to have a smaller free-oxygen supply than spectral type $M$ stars and they show ZrO lines preferentially over the less stable TiO lines seen in M stars. (Snyder and Buhl 1975 originally reported $\mathrm{R}$ Hya as an $\mathrm{S}$ star $\mathrm{SiO}$ source, but the Kukarkin " $S$ " designation is erroneous [P. Keenan, private communication].)

Because no S star has ever shown water emission, it seemed possible that the $\chi \mathrm{Cyg} \mathrm{SiO}$ maser reflected a basic difference in the chemistry of $S$ and $M$ stars. One purpose of this experiment was to look at a substantial number of bright $\mathrm{S}$ stars in both $\mathrm{SiO}$ and $\mathrm{H}_{2} \mathrm{O}$ to see whether $\mathrm{SiO}$ masers are found in such stars and whether $\mathrm{H}_{2} \mathrm{O}$ is ever present. We selected stars which possessed the characteristics shared by other $\mathrm{H}_{2} \mathrm{O}-\mathrm{SiO}$ stars: redness as indicated by their $I-K$ color index and, in the case of Mira variables, an optical variability of at least $5 \mathrm{mag}$. An attempt was also made to choose nearby stars, based on their visual or $K$-magnitude or a kinematic distance estimate.

\section{OBSERVATIONS}

The SiO observations were made with the 36 foot (11 m) telescope of the National Radio Astronomy Observatory, ${ }^{1}$ Kitt Peak, Arizona. The $v=1, J=$

\footnotetext{
1 The National Radio Astronomy Observatory is operated by Associated Universities, Inc., under contract with the National Science Foundation.
}

$1 \rightarrow 0$ transition at $43,122.0 \mathrm{MHz}$ was observed with a mixer-diode receiver whose single sideband temperature was $\sim 1400 \mathrm{~K}$. The $v=1, J=2 \rightarrow 1$ line $(86,243.3 \mathrm{MHz})$ was observed with the cooled mixer receiver whose single sideband system temperature was $\sim 600 \mathrm{~K}$. Spectral information was provided by two filter banks of 256 channels each; the individual filters were $100 \mathrm{kHz}$ in one case, and $250 \mathrm{kHz}$ in the other. Total-power position switching was employed as the observing mode.

Observations of the $J=6_{16} \rightarrow 5_{23}$ rotational line of water vapor $(22,235.08 \mathrm{MHz})$ were made on the 120 foot $(37 \mathrm{~m})$ paraboloid of the Haystack Observatory, ${ }^{2}$ Westford, Massachusetts. The system has a maser front end with a 100 channel autocorrelator providing spectral information. System temperature lay between $100 \mathrm{~K}$ and $200 \mathrm{~K}$, depending on the weather. Total-power position switching was used here, too, with the exception that off-source runs were made to track the same sky path as the on-source runs to prevent adverse effects on the maser gain due to changes in the Earth's magnetic field.

The primary search was conducted at $43 \mathrm{GHz}$ with some search work being done at $86 \mathrm{GHz}$. A total of 83 sources was examined. Near-coincidence with solar right ascension made it necessary to observe some sources with the dome closed to prevent thermal deformation of the antenna. Stars detected in this manner have been so noted.

\section{RESULTS}

Eight new stars have been detected with vibrationally excited $\mathrm{SiO}$ emission, bringing the total of such stars

\footnotetext{
${ }^{2}$ The Haystack Observatory is operated by the Northeast Radio Observatory Corporation with support from the National Science Foundation.
} 
TABLE 1

New Detections

\begin{tabular}{|c|c|c|c|c|c|}
\hline Name & IRC & $\begin{array}{l}\text { Frequency } \\
\quad(\mathrm{GHz})\end{array}$ & $\begin{array}{c}T_{\text {ant }} * \\
(\mathrm{~K})\end{array}$ & $\begin{array}{c}\Delta V \\
\left(\mathrm{~km} \mathrm{~s}^{-1}\right)\end{array}$ & $\begin{array}{l}\text { Velocity } \\
\left(\mathrm{km} \mathrm{s}^{-1}\right)\end{array}$ \\
\hline \multirow{2}{*}{$\begin{array}{l}\text { CIT } 3 \ldots \ldots \ldots \\
\text { W And } \ldots \ldots \ldots\end{array}$} & +10011 & 86 & 1.1 & $\sim 3$ & +8 \\
\hline & +40037 & $\begin{array}{l}43 \\
86\end{array}$ & $\begin{array}{l}0.6 \\
0.7\end{array}$ & & $\begin{array}{l}-34 \\
-33\end{array}$ \\
\hline \multirow[t]{2}{*}{ R Cnc....... } & +10185 & 43 & 2.2 & 3.5 & +19 \\
\hline & & 86 & $2.1,2.0$ & $\sim 2, \sim 3$ & $+19,13.8$ \\
\hline \multirow{2}{*}{ RU Her.... . . . } & +30282 & 43 & 0.9 & 1.8 & -11.5 \\
\hline & & 86 & 1.3 & 2.7 & -11.5 \\
\hline \multirow[t]{3}{*}{ AH Sco........ } & -30282 & $\begin{array}{l}43 \\
86\end{array}$ & $\sim 1.0$ & $\begin{aligned} & 4.9 \\
\sim & 4\end{aligned}$ & $\begin{array}{l}+0.7 \\
\sim-2\end{array}$ \\
\hline & -20424 & 43 & 0.5 & 3.8 & +15.0 \\
\hline & -10529 & 43 & 2.0 & $\sim 1$ & -17.0 \\
\hline \multirow{2}{*}{ T Cep ........ } & & 43 & 2.5 & $\sim 1.5$ & $\begin{array}{l}-19.0 \\
-14\end{array}$ \\
\hline & +70108 & $\begin{array}{l}43 \\
86\end{array}$ & $\begin{array}{l}1.0 \\
1.4\end{array}$ & $\begin{array}{r}2.4 \\
\sim 3.5\end{array}$ & $\begin{array}{l}-1.4 \\
-1.7\end{array}$ \\
\hline
\end{tabular}

to 31. Three are S-type, 15 are oxygen-rich Mira variables, six are semiregular variables, and one is an irregular variable. The remainder are not optically identified. Table 1 gives identification, antenna temperature (corrected to outside the Earth's atmosphere for a lossless antenna), line width, and velocity (LSR) for both frequencies (where appropriate). The lines are, in general, weak, and the error in velocity and line width is, conservatively, $\pm 0.8 \mathrm{~km} \mathrm{~s}^{-1}$. We comment briefly on individual sources.

The infrared source IRC +10011 (Fig. 1) has no counterpart in the General Catalogue of Variable Stars (Kukarkin et al. 1969). It is a highly reddened object with a visual magnitude greater than 20. Zappala et al. (1974) identify it as an oxygen-rich Mira variable on the basis of its 650 -day period, $K$-magnitude (1.63), and infrared spectrophotometry. Its $I-K$ index is 7.60 in the Two-Micron Sky Survey (Neugebauer and Leighton 1969). It has $\mathrm{OH}$ emission at $27 \mathrm{~km} \mathrm{~s}^{-1}$ and

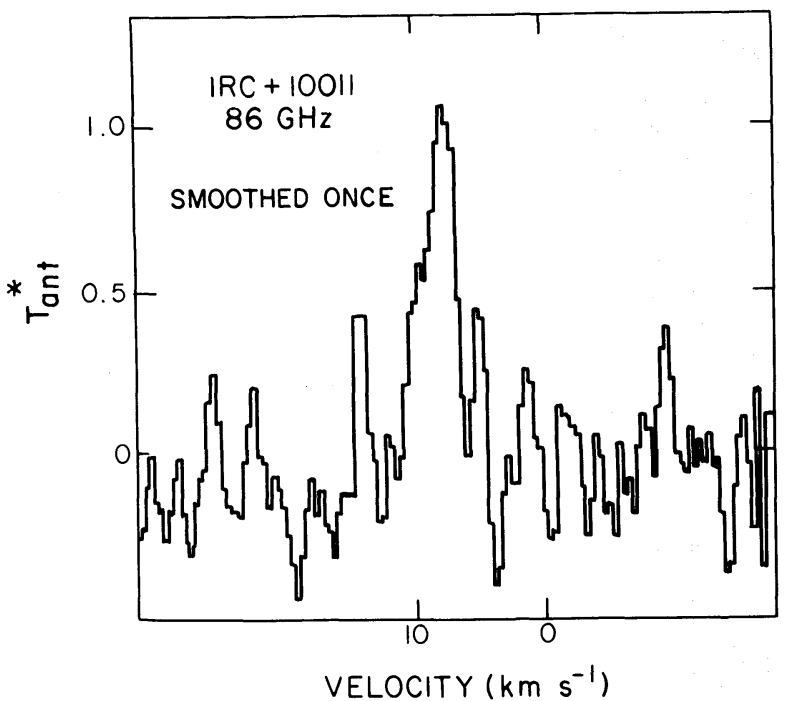

FIG. 1. - The $86 \mathrm{GHz} \mathrm{SiO}(J=2 \rightarrow 1, v=1)$ spectrum of IRC + 10011 (CIT 3).
$-9 \mathrm{~km} \mathrm{~s}^{-1}$ and an $\mathrm{H}_{2} \mathrm{O}$ line at $22 \mathrm{~km} \mathrm{~s}^{-1}$ (Wilson and Barrett 1972; Dickinson 1976). IRC + 10011 was searched in $\mathrm{SiO}$ only at $86 \mathrm{GHz}$.

W And (Fig. 2) is a Mira-type star classed as S6.1e by Kukarkin. W And is the second $\mathrm{S}$ star to show emission from vibrationally excited $\mathrm{SiO}$. W And has been twice examined for $\mathrm{H}_{2} \mathrm{O}$ and has been negative to less than 3 Jy despite the fact that its visual magnitude changes from 6.7 to $14.5 \mathrm{mag}$, its $K$-magnitude is 0.88 , and its $I-K$ index is 5.60 -all three quantities being good indications of possible water emission in oxygen-rich Mira variables. It is also negative in $\mathrm{OH}$ (Wilson and Barrett 1972). We have searched $26 \mathrm{~S}$ stars for $\mathrm{H}_{2} \mathrm{O}$ to a level of, typically, $5 \mathrm{Jy}$. They include: R And, RR And, W And, W Aql, R Cam, T Cam, V Cnc, RS Cnc, S Cas, U Cas, WY Cas, T CMi, T Cep, W Cet, AA Cyg, R Cyg, S Cyg, $\chi$ Cyg, R Gem, T Gem, ST Her, R Lyn, SX Peg, T Sgr, ST Sgr, and S UMa. All are negative.

The star R Cnc (Fig. 3) has two strong features at $86 \mathrm{GHz}$ at $19 \mathrm{~km} \mathrm{~s}^{-1}$ and $13.8 \mathrm{~km} \mathrm{~s}^{-1}$. By contrast, the $43 \mathrm{GHz}$ emission shows only the $19 \mathrm{~km} \mathrm{~s}^{-1}$ clearly; a weak noiselike spike is the only suggestion of the lower velocity feature. $\mathrm{R}$ Cnc is a Mira variable which is quite bright both visually $(6.2 \mathrm{mag})$ and at $2.2 \mu \mathrm{m}$ $(-0.74 \mathrm{mag})$. It is spectral type M6-M8. It is negative in $\mathrm{H}_{2} \mathrm{O}$. Its visual magnitude differential (6.2-11.8) is low; all Miras with water emission show a change of more than 6 mag (Dickinson 1976). It is also negative in $\mathrm{OH}$ at 1612,1665 , and $1667 \mathrm{MHz}$ to about $1 \mathrm{Jy}$ (Wilson and Barrett 1972). This SiO maser was discovered independently by Spencer et al. (1976).

In RU Her (Fig. 4), the sharp, narrow feature at $43 \mathrm{GHz}$ suggests that the wider $86 \mathrm{GHz}$ feature is actually a blend of two features at $-11.5 \mathrm{~km} \mathrm{~s}^{-1}$ and $-10 \mathrm{~km} \mathrm{~s}^{-1}$. A relatively long-period Mira variable (485 days), RU Her is spectral type M6-M7. It has been searched on three different occasions for $\mathrm{OH}$ and $\mathrm{H}_{2} \mathrm{O}$ without success-despite the fact that its infrared and optical properties are typical of such stars.

AH Sco (Fig. 5) is a semiregular variable, M3, recently detected in the water line by Lepine, Paes de Barros, and Gammon (1976). It has an unusually long 

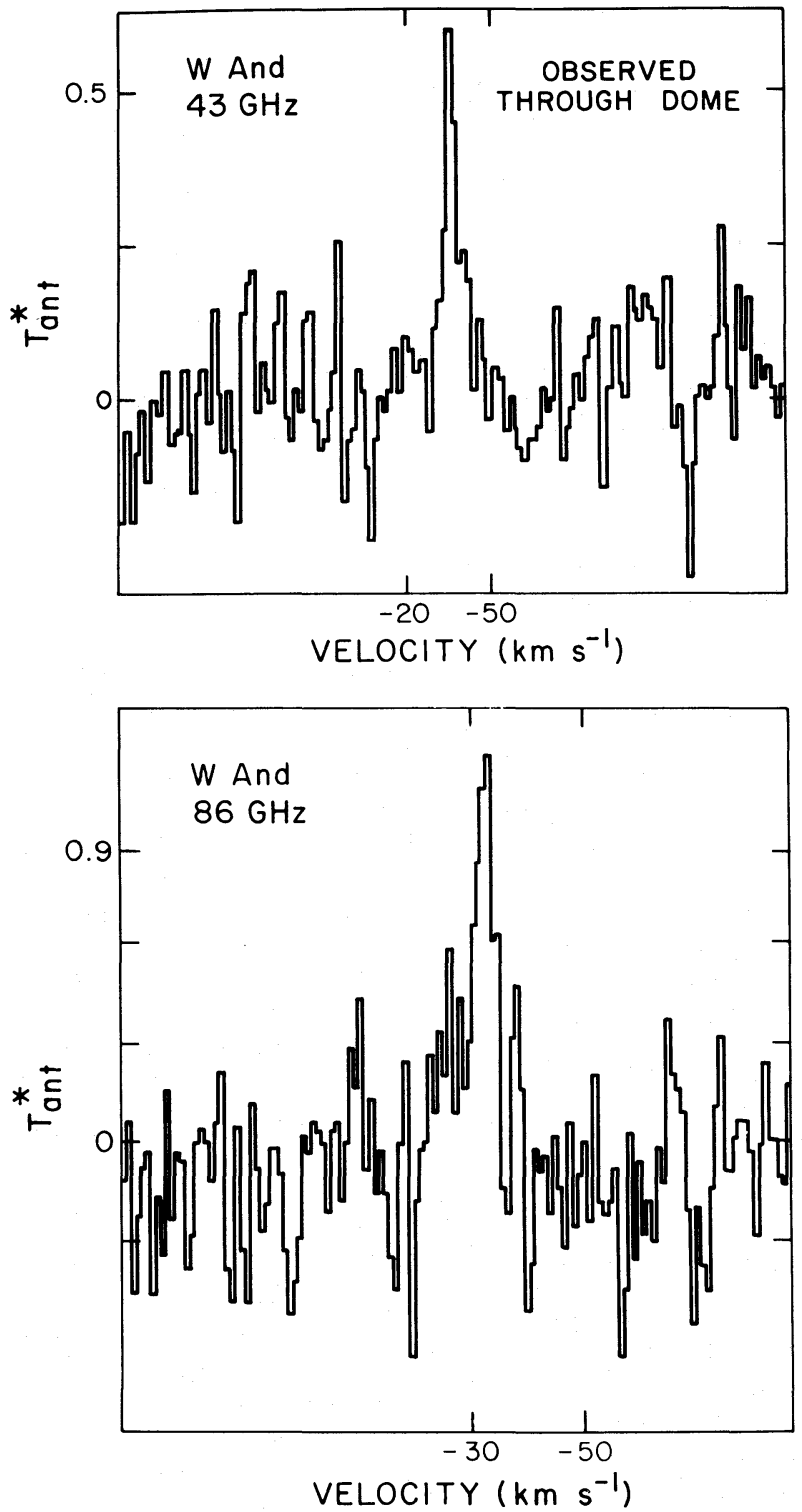

FIG. 2-The $\mathrm{SiO}(v=1)$ rotational spectra of $\mathrm{W}$ And $(\mathrm{IRC}+40037)$ at $43 \mathrm{GHz}(J=1 \rightarrow 0)$ and $86 \mathrm{GHz}(J=$ $2 \rightarrow 1)$. To convert to heliocentric velocity, add $-0.2 \mathrm{~km} \mathrm{~s}^{-1}$.

period, 714 days. The $43 \mathrm{GHz} \mathrm{SiO}$ line is apparently a single feature. It appears to be present also at $86 \mathrm{GHz}$, although time did not permit us to integrate down the noise. AH Sco has a $K$-magnitude of 0.32 and an $I-K$ color index of 3.99. A. Baudry (private communication) has recently detected a double-peaked $\mathrm{OH}$ spectrum from this star. The velocity separation is $\sim 40 \mathrm{~km} \mathrm{~s}^{-1}$, which confirms nicely the correlation between period and radial-velocity pattern (Dickinson, Kollberg, and Yngvesson 1975).

IRC -20424 (Fig. 6) has a substantial infrared excess from circumstellar dust which is probably also responsible for obscuring the optical object within. Its $I-K$ index is 6.31 ; its $K$-magnitude (0.90) makes it a
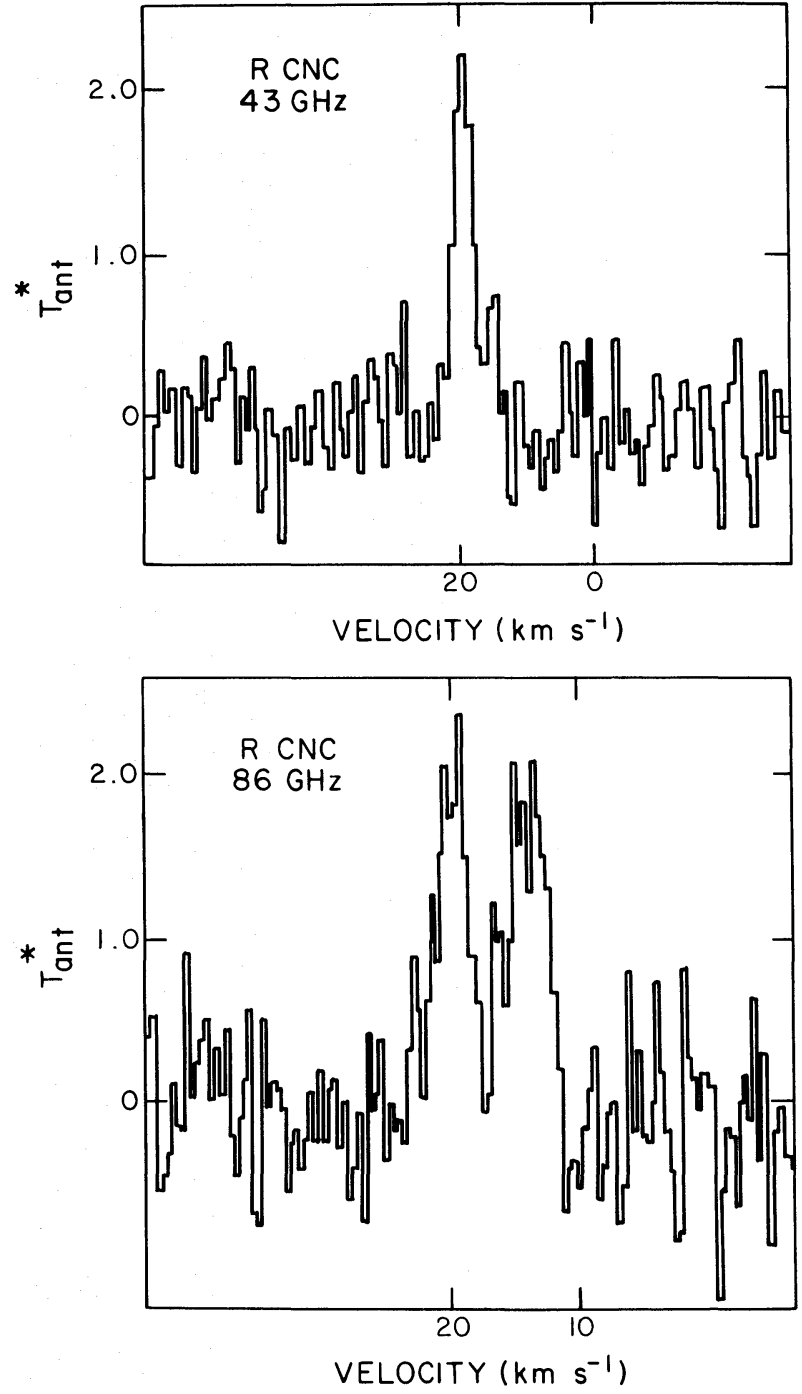

FIG. 3.-The $\mathrm{SiO}(v=1)$ rotational spectra of $\mathrm{R} \mathrm{Cnc}$ $($ IRC +10185$)$ at $43 \mathrm{GHz}(J=1 \rightarrow 0)$ and $86 \mathrm{GHz}(J=$ $2 \rightarrow 1)$. To convert to heliocentric velocity, add $12.2 \mathrm{~km} \mathrm{~s}^{-1}$.

relatively bright infrared source. Water emission is seen at $18.8 \mathrm{~km} \mathrm{~s}^{-1}$, and the double group of $\mathrm{OH}$ emission features, characteristic of infrared stars, is also present (Dickinson, Bechis, and Barrett 1973; Wilson et al. 1972).

IRC -10529 is a type $\mathrm{IIb} \mathrm{OH}$ source, negative in water emission (Wilson and Barrett 1972; Dickinson 1976). It exhibits the characteristic double-peaked $\mathrm{OH}$ spectrum but has, unexpectedly, an intermediate velocity component at $1665 \mathrm{MHz}$ and $1667 \mathrm{MHz}$ which is $80 \%$ left circularly polarized. The two $\mathrm{SiO}$ emission features in Figure 7 bracket this feature. Its $K$ magnitude is 2.65 , and it is highly reddened with an $I-K$ of 6.77 .

T Cep shows a single feature at $43 \mathrm{GHz}$ (Fig. 8). The higher frequency spectrum suggests there may be more than one peak, but the signal-to-noise ratio is too poor 

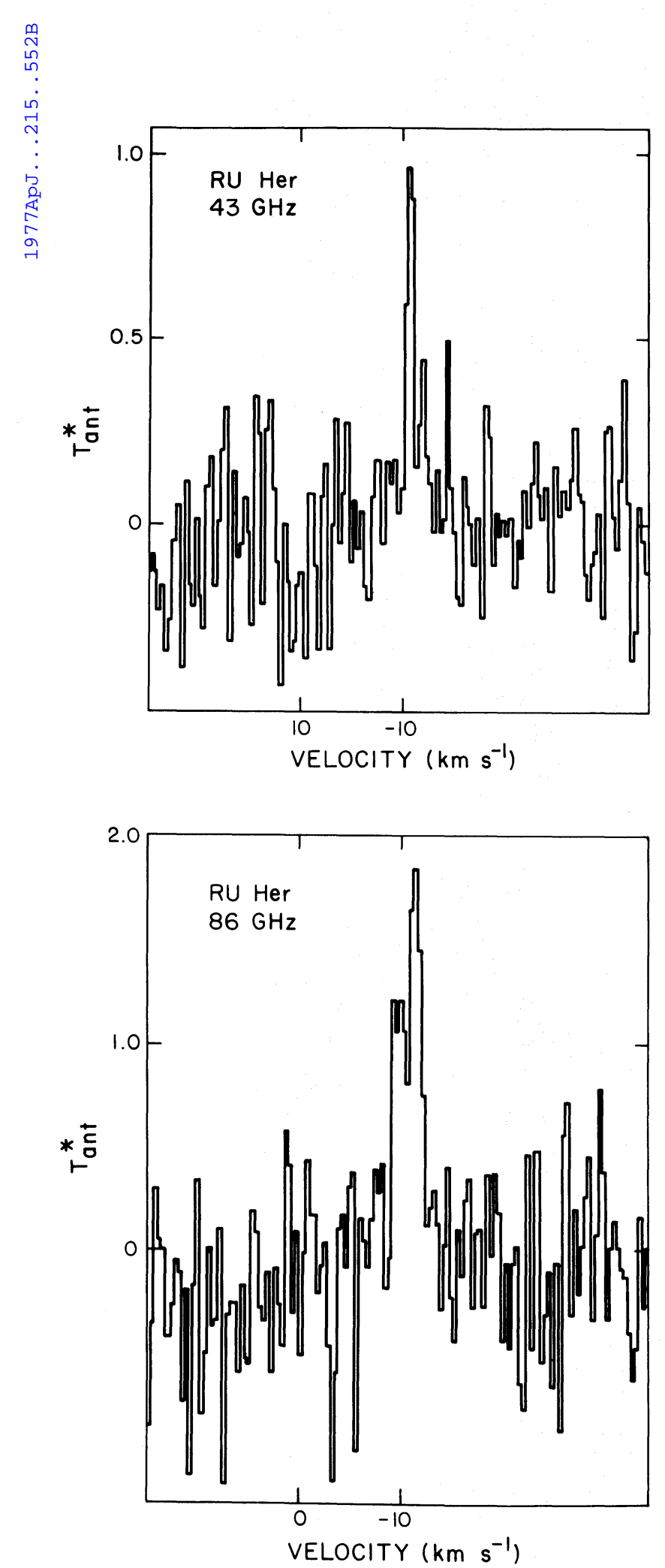

FIG. 4.-The $\mathrm{SiO}(v=1)$ rotational spectra of RU Her $(\mathrm{IRC}+30282)$ at $43 \mathrm{GHz}(J=1 \rightarrow 0)$ and $86 \mathrm{GHz}(J=$ $2 \rightarrow 1)$. To convert to heliocentric velocity, add $-18.0 \mathrm{~km} \mathrm{~s}^{-1}$.
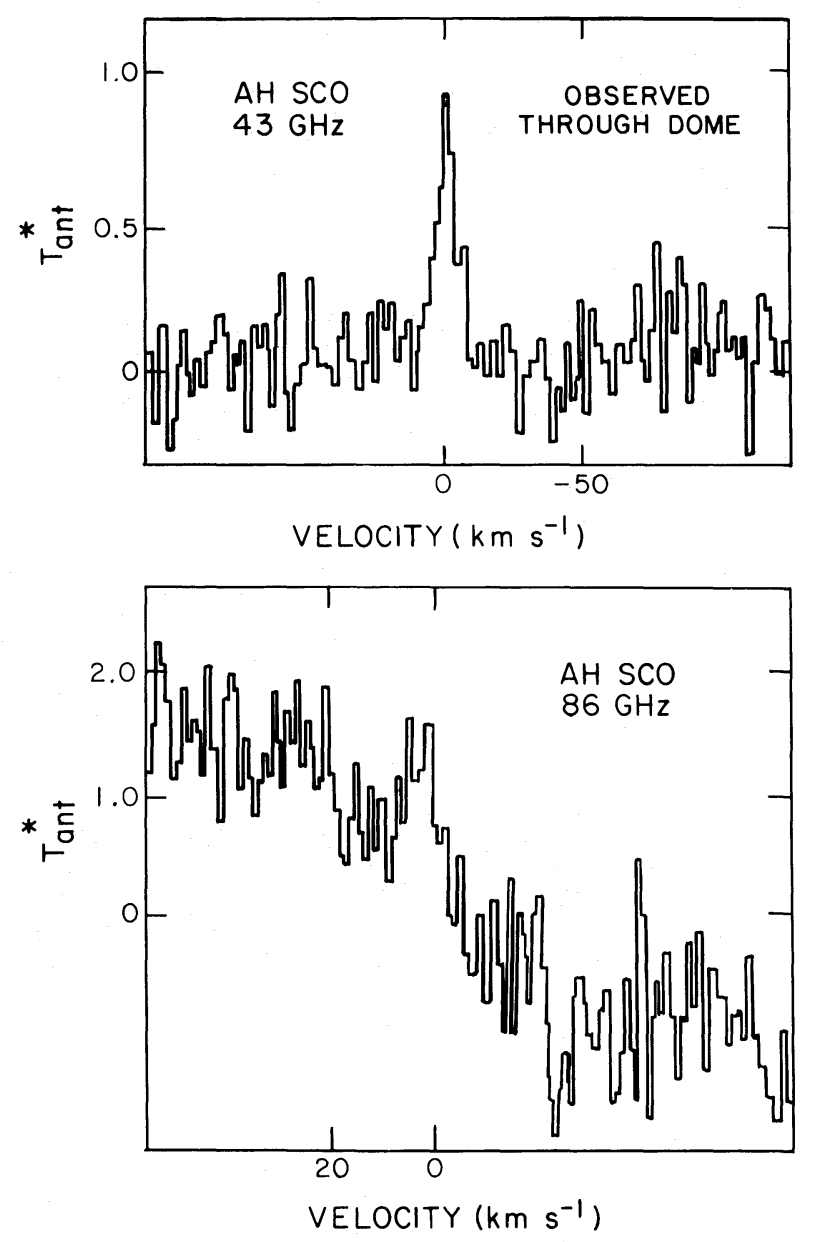

FIG. 5. - The $\mathrm{SiO}(v=1)$ rotational spectra of $\mathrm{AH}$ Sco (IRC - 30282) at $43 \mathrm{GHz}(J=1 \rightarrow 0)$ and $86 \mathrm{GHz}(J=$ $2 \rightarrow 1)$. To convert to heliocentric velocity, add $-7.5 \mathrm{~km} \mathrm{~s}^{-1}$.

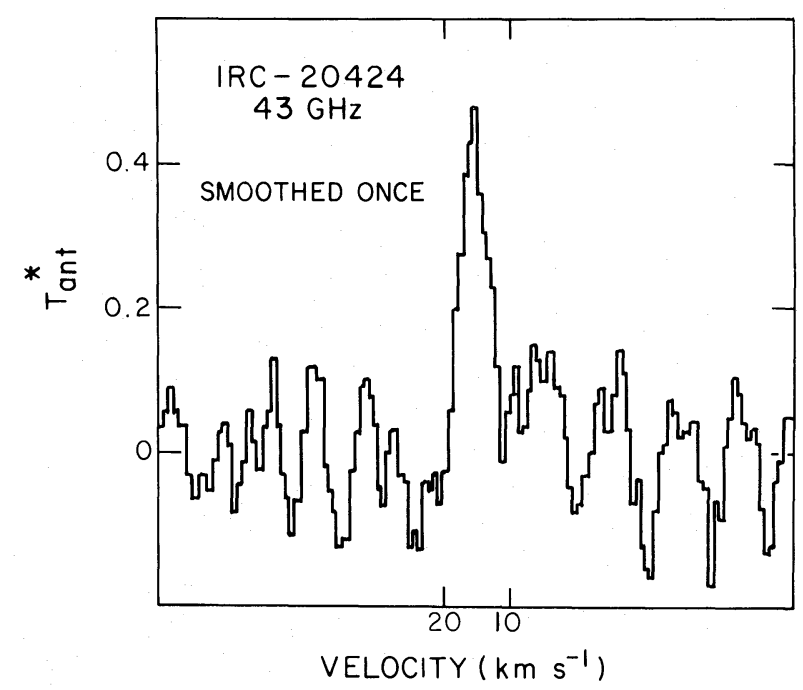

FIG. 6.-The $43 \mathrm{GHz} \mathrm{SiO}(J=1 \rightarrow 0, v=1)$ spectrum of IRC -20424 


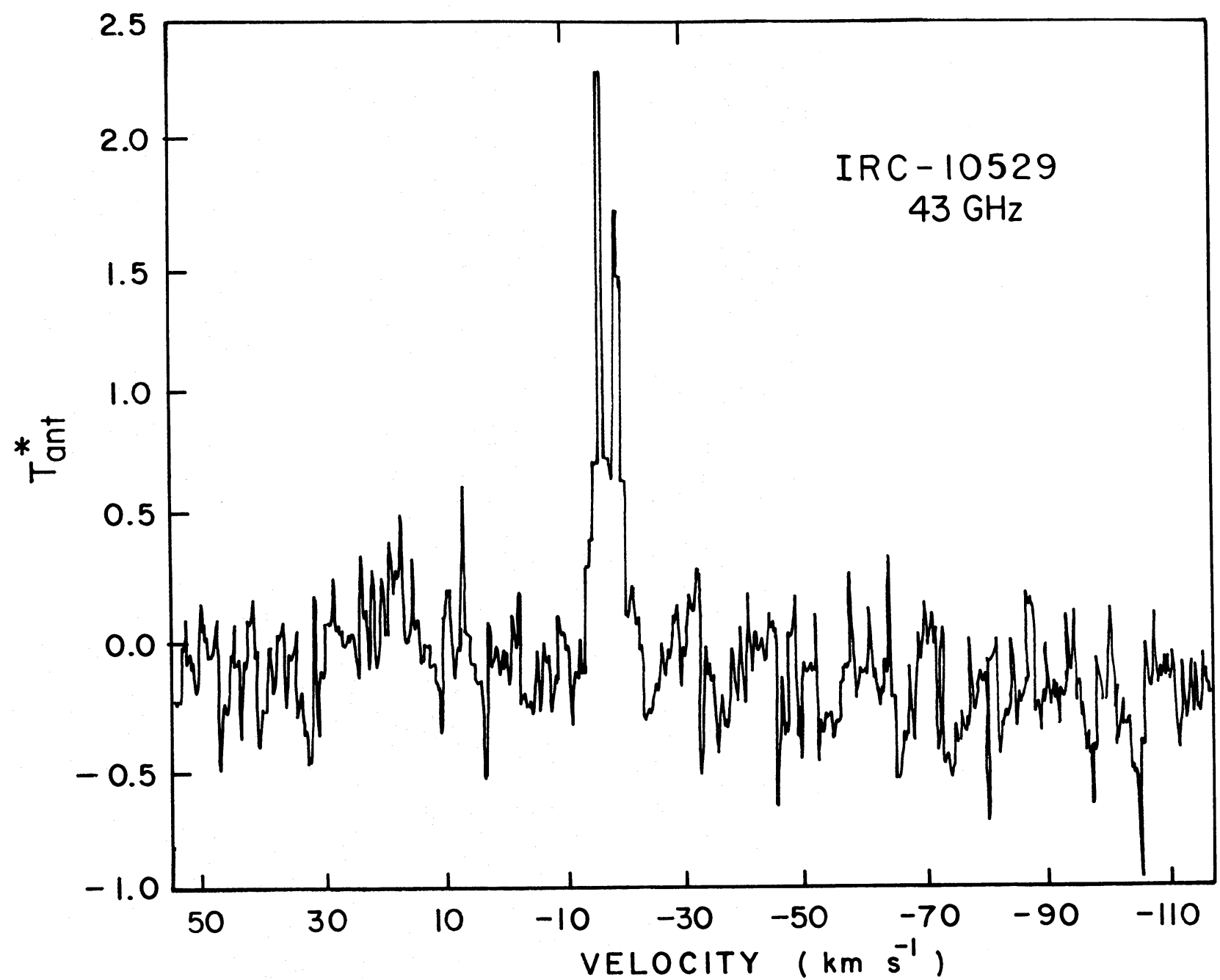

FIG. 7.-The $43 \mathrm{GHz} \mathrm{SiO}(J=1 \rightarrow 0, v=1)$ spectrum of IRC -10529

to tell with certainty. It has been searched for water on three different occasions without success. It is an extremely bright infrared object $(K=-1.56)$, and its visual change goes from 5.4 to $11.0 \mathrm{mag}$. Listed as M5e-M9e by Kukarkin et al. (1969), it was classified as an S star by Spinrad and Newburn (1965), but it is really between $M$ and $S$ on the basis of subsequent spectra (Keenan, private communication). This source was found independently by Spencer et al. (1976).

Concerning NGC 2264 (Fig. 9), while completing some calibration work on the ground vibrational state, $J=2 \rightarrow 1$ line of $\mathrm{SiO}(86,846.85 \mathrm{MHz})$, we observed a new emission line, the first ground-state $\mathrm{SiO}$ emission from a molecular cloud seen outside of Sgr B2 and Orion A. The telescope was pointed at $\alpha_{1950}=$ $6^{\mathrm{h}} 38^{\mathrm{m}} 28 \mathrm{~s} 4, \delta_{1950}=9^{\circ} 32^{\prime} 12^{\prime \prime}, 55^{\prime \prime}$ from the position of Allen's near-infrared source (Allen 1972). The line is $2 \mathrm{~km} \mathrm{~s}^{-1}$ broad and has a peculiar velocity of about $7.5 \mathrm{~km} \mathrm{~s}^{-1}$. This is one-half to one-third the line width of molecular emission lines in NGC 2264. NGC 2264 has not yet been searched for vibrationally excited $\mathrm{SiO}$ emission and clearly warrants additional work.

\section{a) Infrared $\mathrm{SiO}$ Study}

Since most theoretical models of maser emission invoke some form of infrared pumping (Mather and Litvak 1974; Kwan and Scoville 1974), we have investigated the relation of $\mathrm{SiO} v=1, J=1 \rightarrow 0$ flux with 4.8 and $10 \mu \mathrm{m}$ flux for all known $\mathrm{SiO}$ maser sources. Such pumping could occur in $\mathrm{SiO}$ via the lowest vibrational transition at $\sim 8 \mu \mathrm{m}$ or its first overtone at $\sim 4 \mu \mathrm{m}$. We note that, subject to infrared and $\mathrm{SiO}$ variability, there is a rough correlation in the data plotted in Figure 10. The scatter in the infrared data in Figure 7 is on the order of the variability observed by Forrest, Gillett, and Stein (1975). We plot peak SiO flux (W m-2 $\left.\mathrm{Hz}^{-1}\right)$ because the narrowness of the maser lines renders them unresolved by the $100 \mathrm{kHz}$ filters in many cases. This causes the points in Figure 10 for sources with exceptionally narrow lines to appear to the left of their true position in the diagram. The equal photon of $100 \%$ efficiency line drawn in Figure 10 is calculated by assuming equal fractional line widths for the infrared pumping line 

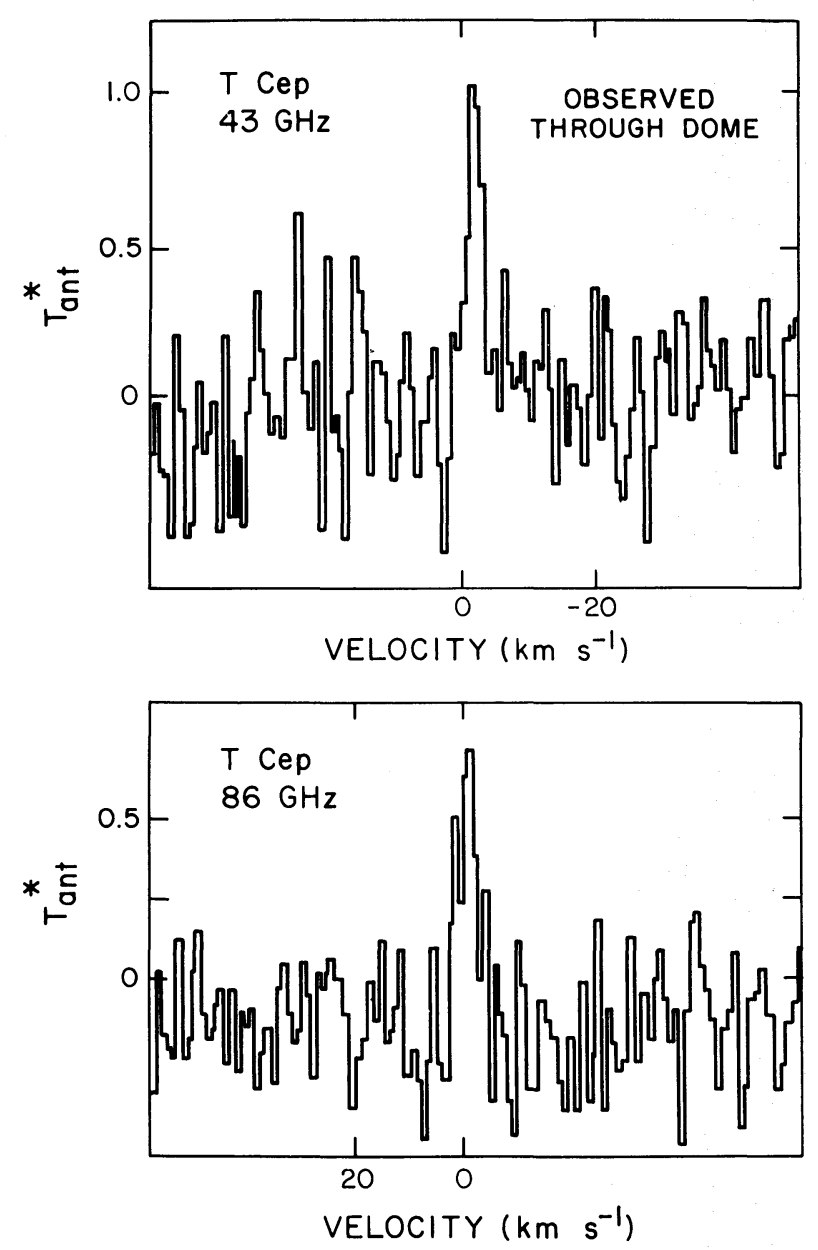

FIG. 8.-The $\mathrm{SiO}(v=1)$ rotational spectra of $\mathrm{T}$ Cep $(\mathrm{IRC}+70168)$ at $43 \mathrm{GHz}(J=1 \rightarrow 0)$ and $86 \mathrm{GHz}(J=$ $2 \rightarrow 1)$. To convert to heliocentric velocity, add $-13.7 \mathrm{~km} \mathrm{~s}^{-1}$.

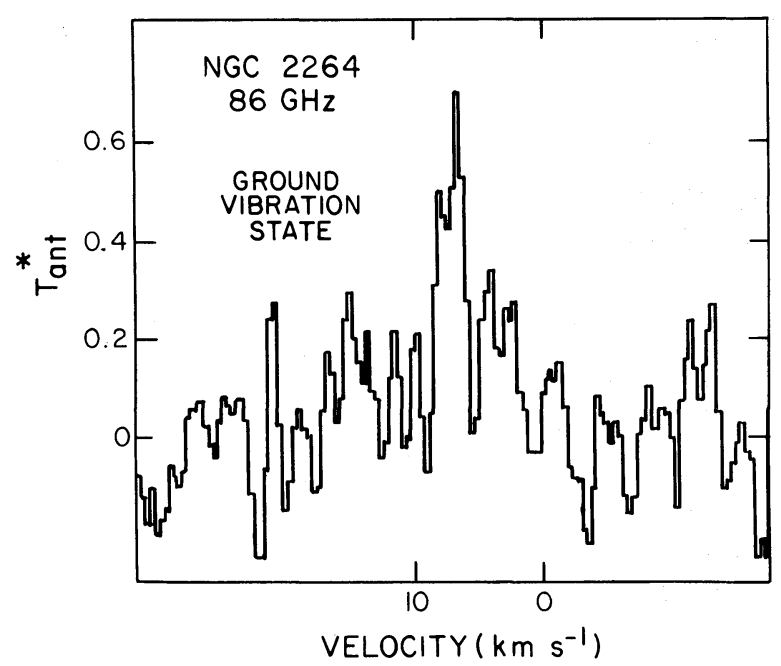

FIG. 9. - The ground-state $(v=0) \mathrm{SiO}$ rotational spectrum of NGC 2264 at $86 \mathrm{GHz}(J=2 \rightarrow 1)$. and the $43.1 \mathrm{GHz}$ maser line. Because the maser lines may be narrower than the thermal Doppler width, the $100 \%$ efficiency line shown is the most stringent that can be chosen as a test of IR pumping. Even more striking than the rough correlation discussed above is the abrupt cutoff of sources just short of the $100 \%$ efficiency line. While this type of comparison does not prove the existence of infrared pumping, it provides a plausible consistency check. Finally, we note that Orion A is the only source which violates this check. The deep $10 \mu \mathrm{m}$ silicate absorption in Orion suggests that there is much more dust present than exists in the shells about the $\mathrm{SiO}$ maser stars; thus the $4.8 \mu \mathrm{m}$ flux could be heavily attenuated, causing Orion to fall to the right of the " $100 \%$ efficiency" line. We call attention to the fact, however, that a number of Orion-like objects have been observed (i.e., Mon R2, S140 IR, etc.) and show no $\mathrm{SiO}$ maser emission. Orion appears to be a unique object, and infrared pumping, conceivably, does not apply.

\section{DISCUSSION}

Traditionally, $\mathrm{S}$ stars are those long-period variables where $\mathrm{ZrO}$ lines become prominent at the expense of the TiO emission usually seen in spectral type M stars. $\mathrm{S}$ stars have a higher $\mathrm{C} / \mathrm{O}$ ratio than the oxygen-rich $\mathrm{M}$ stars. Scalo (1974) has done detailed calculations on molecular abundances for varying $\mathrm{C} / \mathrm{O}$ ratios: we reproduce, for convenience, the appropriate figure from his paper (Fig. 11). The abscissa can be thought of as grading from $M$ stars on the left, to $S$ stars near $\mathrm{C} / \mathrm{O}=0.9$. Further increase in the $\mathrm{C} / \mathrm{O}$ ratio leads one to the domain of carbon stars. (There may, in fact, be an evolutionary sequence in long-period variables from $M$ through $S$ to $C$ in spectral type [Scalo 1974].) While the $\mathrm{H}_{2} \mathrm{O} / \mathrm{SiO}$ ratio remains roughly constant over a significant range of $\mathrm{C} / \mathrm{O}$ values, the $\mathrm{SiO}$ eventually dominates, being an order of magnitude larger than $\mathrm{H}_{2} \mathrm{O}$ for a short while near $\mathrm{C} / \mathrm{O}=0.95$. This is consistent with our ability to detect a limited number of $\mathrm{SiO}$ masers in $\mathrm{S}$ stars but no $\mathrm{H}_{2} \mathrm{O}$ masers.

Table 2 lists 76 sources searched at $43 \mathrm{GHz}$ for excited vibrational state $\mathrm{SiO}$ emission. Upper limits varied somewhat, but were, typically, $0.5 \mathrm{~K}$ in the $250 \mathrm{kHz}$ filters and $0.75 \mathrm{~K}$ in the $100 \mathrm{kHz}$ filters. Distance was an important factor in detectability. At least five of the SiO stars, W And, R Cnc, RU Her, $\mathrm{T}$ Cep, and IRC +10011 , are thought to be within 500 pc. For convenience, Table 3 lists all SiO maser stars known at this writing.

The three water-emission stars have properties as characterized by Snyder and Buhl (1975). IRC + 10011 is a type $\mathrm{IIb} \mathrm{OH}$ source, the $\mathrm{SiO}$ velocity falling near the high-velocity OH peak. IRC -20424 is a type I OH source with its $\mathrm{SiO}$ emission about $3 \mathrm{~km} \mathrm{~s}^{-1}$ less than the high-velocity $\mathrm{OH}$. Of the five $\mathrm{SiO}$ stars which show neither $\mathrm{OH}$ nor $\mathrm{H}_{2} \mathrm{O}$, two are $\mathrm{S}$ stars, $\chi \mathrm{Cyg}$ and W And, and may be expected to be negative for the reasons previously discussed. $\mathrm{R} \mathrm{Cnc}$, as noted, lacks the large differential magnitude change characteristic of $\mathrm{OH} / \mathrm{H}_{2} \mathrm{O}$ sources. The remaining star, $\mathrm{RU}$ Her, is 


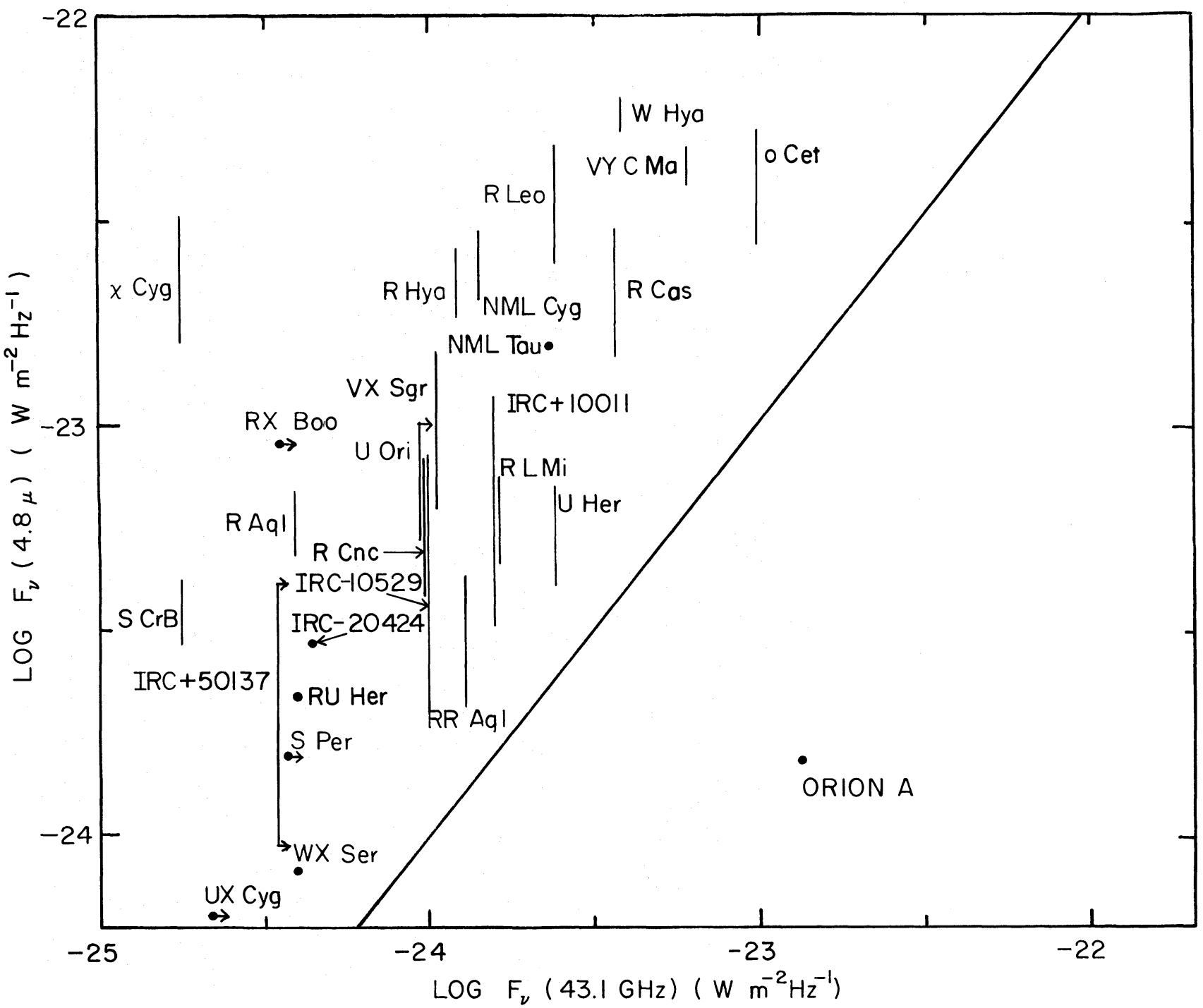

Fig. 10.- The peak flux density at $43.1 \mathrm{GHz}$ is plotted versus the $4.8 \mu \mathrm{m}$ flux density for all known SiO masers. The range of infrared variability is used where available. The $45^{\circ}$ line corresponds to equal number of photons at $43.1 \mathrm{GHz}$ and $4.8 \mu \mathrm{m}$. All 43.1 GHz data (except for sources reported herein) are from Snyder and Buhl 1975. The infrared data are from Becklin et al. 1973, Forrest et al. 1975, Gehrz and Woolf 1971, Hyland et al. 1972, and Wilson et al. 1972.

still a puzzle; it possesses all the indicators of $\mathrm{OH}$ and $\mathrm{H}_{2} \mathrm{O}$ maser stars, but has, as yet, not rewarded us with a detection.

We note in closing that, since publication of Snyder and Buhl (1975) and Kaifu, Buhl, and Snyder (1975), WX Ser has shown $22 \mathrm{GHz}$ water emission and S Per has been seen in both $\mathrm{OH}$ and $\mathrm{H}_{2} \mathrm{O}$ (Dickinson, Kollberg, and Yngvesson 1975; Dickinson 1976). They join the 15 other $\mathrm{SiO}$ stars which also show $\mathrm{OH}$ and $\mathrm{H}_{2} \mathrm{O}$. RX Boo shows $\mathrm{SiO}$ and $\mathrm{H}_{2} \mathrm{O}$ but not OH. IRC +50137 has $\mathrm{SiO}$ and $\mathrm{OH}$ but not $\mathrm{H}_{2} \mathrm{O}$; this may be only a sensitivity problem, as the $\mathrm{SiO}$ emission is marginal and the $K$-magnitude is 2.72 -very dim.

We have also looked at a number of objects which resemble the Becklin-Neugebauer object in Orion:
W33 A, W33 B, Onsala 1, NGC 7538, Mon R2, and S140 IR. All are strong near-infrared sources without radio continuum. All are negative in $\mathrm{SiO}$ excited state emission, suggesting that the BN object is unique and may not be properly classifiable with these other sources.

We wish to thank Drs. Pat Thaddeus and J. H. Davis for assistance with some of the observations and Dr. Tom Barnes for making available his compilation of radial velocities and kinematic distances of late-type stars. G. N. B. was supported in part by the National Science Foundation under grant MPS 05070-76. 


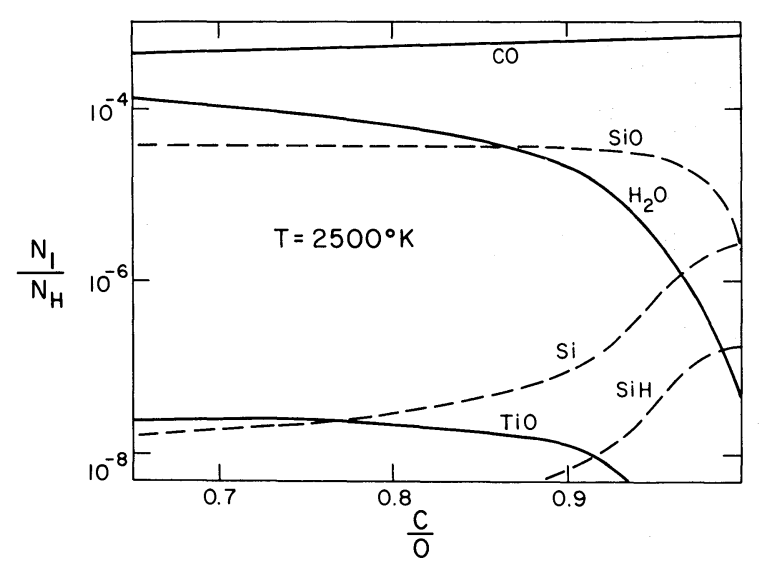

FIG. 11.- Variation with $\mathrm{C} / \mathrm{O}$ of the concentration of observable molecules relative to hydrogen for a temperature of $2500 \mathrm{~K}$. The oxygen abundance is $7 \times 10^{-4}$ and the gas pressure is $10^{3}$ dynes $\mathrm{cm}^{-2}$.
TABLE 2

Negative Results (43 GHz)

\begin{tabular}{lll}
\hline \hline R And & $\chi$ CrB & S UMa \\
$\chi$ And & R Crt & T UMa \\
RR And & AA Cyg & U UMi \\
RW And & Z Del & R Vir \\
$\chi$ Aqr & R Gem & S Vir \\
W Aql & T Gem & U Vir \\
R Aur & S Her & RT Vir \\
U Aur & T Hya & AFCRL 618 \\
TV Aur & $\chi$ Hya & AFCRL 19 \\
R Boo & R Lyn & W 33 A (IR) \\
R Cam & V Mon & W 33 B \\
T Cam & BD Mon & HD 11979 \\
V Cnc & FU Mon & Onsala 1 \\
W Cnc & R Oph & NGC 7538 \\
S CMi & $\chi$ Oph & IRC +30292 \\
V CMi & RZ Per & Mon R2 \\
R CVn & Z Pup & S140 IR \\
U CVn & T Sgr & OH 21.5+0.5 \\
Y CVn & ST Sgr & OH 26.5+0.6 \\
S Cas & V745 Sgr & OH 30.1-0.2 \\
U Cas & RR Sco & OH 30.1-0.7 \\
Y Cas & ST Sco & OH 32.8-0.3 \\
MY Cep & V407 Sco & OH 45.5+0.1 \\
R Com & R Ser & OH 231.4+4.2 \\
T CrB & EI Tau & \\
W CrB & R UMa & \\
\hline
\end{tabular}

TABLE 3

A Catalog of SiO Maser Stars

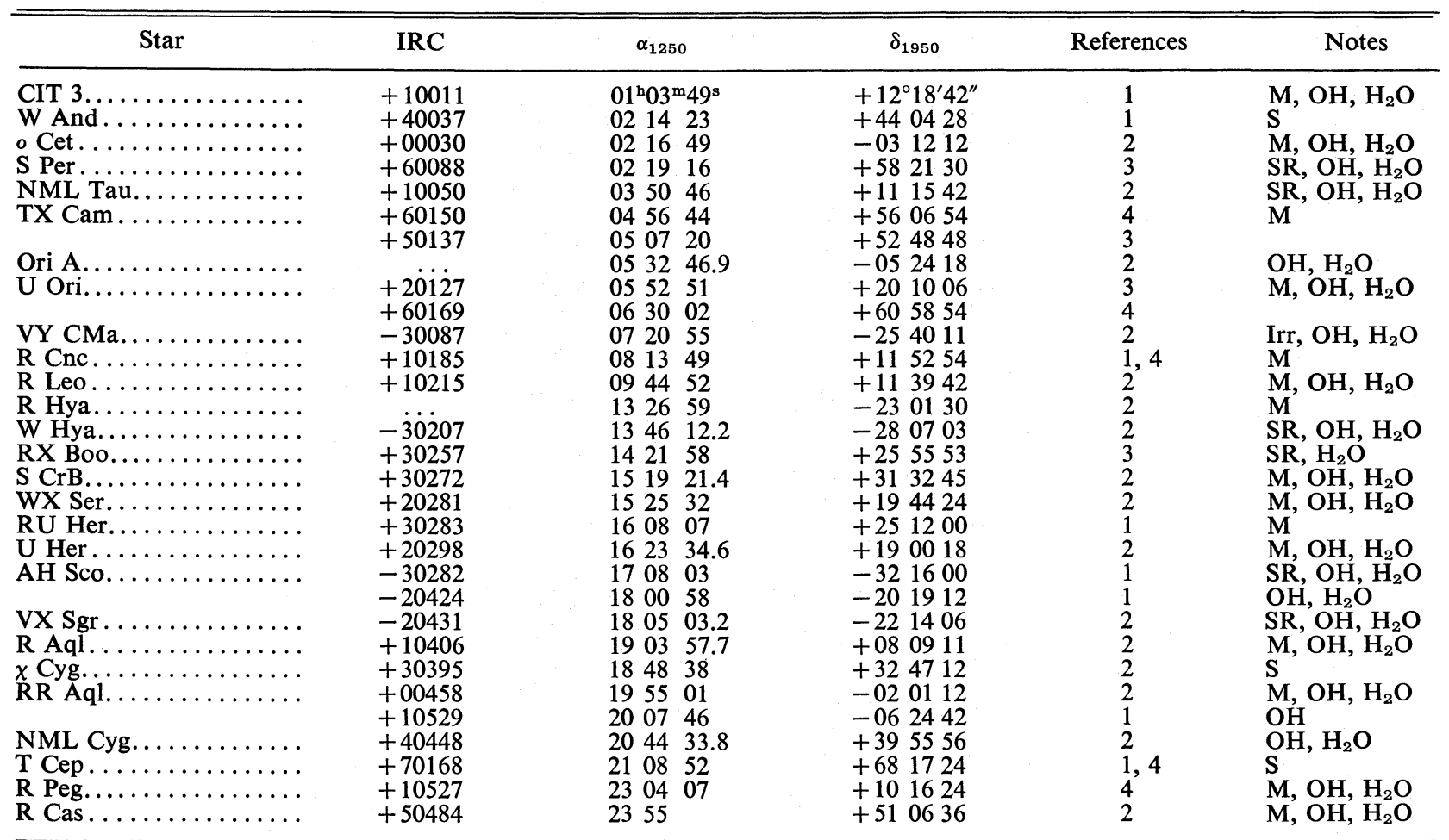

REFERENCES.-1, this paper and Dickinson and Blair 1976; 2, Snyder and Buhl 1975; 3, Kaifu, Buhl, and Snyder 1975; 4, Spencer et al. 1976.

Notes.-M, oxygen-rich Mira variable; S, S-type Mira; SR, semiregular variable; $\mathrm{H}_{2} \mathrm{O}$, water emission; Irr, irregular variable. 


\section{BLAIR AND DICKINSON}

\section{REFERENCES}

Allen, D. A. 1972, Ap. J. (Letters), 172, L55.

Becklin, E. E., Neugebauer, G., and Wynn-Williams, C. G. 1973, Ap. J. (Letters), 182, L7.

Dickinson, D. F. 1976, Ap. J. Suppl., 30, 259.

Dickinson, D. F., Bechis, K. B., and Barrett, A. H. 1973, Ap. J., 180, 831.

Dickinson, D. F., and Blair, G. N. 1976, Bull. $A A S$, 8, 347.

Dickinson, D. F., Kollberg, E., and Yngvesson, S. 1975, Ap. J., 199, 131

Forrest, W. J., Gillett, F. C., and Stein, W. A. 1975, Ap. J., 195,423

Gehrz, R. D., and Woolf, N. J. 1971, Ap. J., 165, 285.

Hyland, A. R., Becklin, E. E., Frogel, J. A., and Neugebauer, G. 1972, Astr. Ap., 16, 204.

Kaifu, N., Buhl, D., and Snyder, L. E. 1975, Ap. J., 195, 359.

Kukarkin, B. V., Kholopov, P. N., Efremov, Yu. N., Kukarkina, N.'P., Kurochkiu, N. E., Medvedeva, G. I., Perova, N. B., Fedorovich, V. P., and Frolov, M. S. 1969 , General Catalogue of Variable Stars (3d ed.; Moscow:

Astronomical Council, Academy of Sciences, USSR).
Kwan, J., and Scoville, N. 1974, Ap. J. (Letters), 194, L97. Lepine, J. R. D., Paes de Barros, M. H., and Gammon, R. 1976, Astr. Ap., in press.

Mather, J. C., and Litvak, M. M. 1974, Bull. AAS, 6, 487-488. Neugebauer, G., and Leighton, R. B. 1969, Two-Micron Sky Survey (NASA N69-37993)

Scalo, J. 1974, Ap. J., 194, 361.

Snyder, L. E., and Buhl, D. 1974, Ap. J. (Letters), 189, L31. -. 1975, Ap. J., 197, 329.

Spencer, J. H., Schwartz, P. R., Bologna, J. M., and Waak, J. A. 1976, Bull. $A A S, 8,372$.

Spinrad, H., and Newburn, R. L. 1965, Ap. J., 141, 965.

Wilson, W. J., and Barrett, A. H. 1972, Astr. Ap., 17, 385.

Wilson, W. J., Schwartz, P. R., Neugebauer, G., Harvey, P. M., and Becklin, E. E. 1972, Ap. J., 177, 523 .

Zappala, R. R., Becklin, E. E., Matthews, K., and Neugebauer, G. 1974, Ap. J., 192, 109.

Note added in proof.--IRC +10011 has subsequently shown $43 \mathrm{GHz}$ excited state $\mathrm{SiO}$ emission at $\sim 28 \mathrm{~km} \mathrm{~s}^{-1}$, in agreement with the high-velocity $\mathrm{OH}$ feature.

GuY N. BlaIR: The Netherlands Foundation for Radio Astronomy, Dwingeloo, The Netherlands

Dale F. Dickinson: Center for Astrophysics, 60 Garden Street, Cambridge, MA 02138 